\title{
Implications of extraterrestrial material on the origin of life
}

\author{
Matthew A. Pasek \\ School of Geosciences, University of South Florida, \\ 4202 E. Fowler Ave, NES 204, Tampa, FL \\ email: mpasek@usf.edu
}

\begin{abstract}
Meteoritic organic material may provide the best perspective on prebiotic chemistry. Meteorites have also been invoked as a source of prebiotic material. This study suggests a caveat to extraterrestrial organic delivery: that prebiotic meteoritic organics were too dilute to promote prebiotic reactions. However, meteoritic material provides building material for endogenous synthesis of prebiotic molecules, such as by hydrolysis of extraterrestrial organic tars, and corrosion of phosphide minerals.
\end{abstract}

Keywords. astrobiology, astrochemistry

\section{Introduction}

The origin of life ultimately relied on the prebiotic chemicals that were present on planetary surfaces. The endogenous production of organic molecules, for instance via the Miller-Urey synthesis (Miller 1953) was assumed to have provided the organic inventory necessary for prebiotic chemistry. However, our current understanding of the geochemical environment of the early earth has changed since these original experiments. The composition of the early atmosphere was likely more oxidizing, and when these experiments are performed under more oxizing conditions, the yield correspondingly diminishes (e.g., Cleaves et al. 2008). The delivery of extraterrestrial organic materials provides an alternative to endogenous synthesis of organic compounds on planetary surfaces. Meteorites and other extraterrestrial material have thus been invoked as a potential or perhaps the principal source of organics to the early earth.

Although meteorites certainly deliver organic materials to planetary surfaces, an open question remains: was the amount delivered by extraterrestrial material sufficient for prebiotic evolution? At present, meteorites, especially those that are rich in organic constituents, are uncommon falls to the earth's surface. Hence this question really supposes two parts: how much could have accumulated, and how much was needed?

\section{How much accumulated?}

Accumulation of extraterrestrial organic material requires survival of the organic compounds during delivery. The likelihood of organic survival is dependent on the way energy is lost during descent through the atmosphere, and changes depending on the size regimes of extraterrestrial material. Broadly, extraterrestrial material consists of three different size fractions: a microscopic fraction including interplanetary dust particles (IDPs) and micrometeorites, a macroscopic fraction comprised of meteorites, and a mega-scale fraction consisting of comets and bolides. The primary distinguishing feature between these three is how they lose energy on atmospheric entry. Small objects, such as IDPs, lose 
energy primarily by radiation, meteorites lose energy by ablation, and bolides do not lose enough energy to prevent explosive impact or airburst.

The energy that each $\mathrm{kg}$ of extraterrestrial material must lose to reach the surface of the earth is roughly equal to at least $V_{e s c}^{2} / 2$, or about $60 \mathrm{MJ} / \mathrm{kg}$, where $V_{\text {esc }}$ is the escape velocity of the earth. In contrast, the energy required to break a $\mathrm{C}-\mathrm{H}$ bond is about $100 \mathrm{kcal} / \mathrm{mol}$, or about $30 \mathrm{MJ} / \mathrm{kg}$. Thus the loss of energy is critical to organic delivery to the earth's surface, especially if the organics are to remain intact. This argument, although very simple, suggests that organic survival during a large impact would be minimal. Indeed, although extraterrestrial organics have been suggested to co-occur with large impacts (Zhao and Bada 1989), these findings have instead been proposed to be linked to delivery by cometary meteor showers before and after the impact (Zahnle and Grinspoon 1990).

Small objects comprise the majority of mass that falls to the earth (Love and Brownlee 1993). These objects lose most of their energy by radiation and ablation. Since radiative energy loss is governed by $\sigma T^{4} \times S A$, smaller objects (with larger surface areas per unit mass) can lose heat quickly by radiation. The timescale of radiative energy loss should be compared to the decomposition timescales of organics. Since, as of yet, few individual organic molecules have been extracted intact from IDPs, a direct check on the stability of organics during atmospheric entry is not yet possible. The thermal decomposition timescales for amino acids have been measured by Yablokov et al. (2013) and are compared to radiative cooling timescales for micrometeorites in Figure 1. In most cases, a higher temperature means that energy dissipates quicker (and slows the object down), but also degrades organics. A happy medium between these two is required to deliver organics efficiently from extraterrestrial microscopic objects: heat dissipation should be rapid enough to decelerate the object, but cool enough to preserve the organics.

Returning to the question at the beginning of this section, the amount of extraterrestrial material that reached the surface of the earth can be estimated using modern extraterrestrial material. Current estimates of the amount of extraterrestrial material that fall each year are about $3 \times 10^{7} \mathrm{~kg}$ (Love and Brownlee 1993). The earth presumably experienced a higher flux in its earlier history, perhaps at a $1000 \times$ increase. Larger impacts were also more common. By modifying the flux calculations of Pasek and Lauretta (2008) to account for the lower total material flux (no longer estimated at $10^{5} \times$ the current day flux- Bottke et al. 2012), the total amount of carbon delivered to the earth could have been $0.1 \mathrm{~kg} / \mathrm{km}^{2}$ per year by IDPs and micrometeorites (assuming little change to organics during atmospheric entry), with meteorite strewn fields perhaps increasing this by a factor of 10 . Note that this is the total mass of soluble organic carbon species, and individual compounds will be considerably lower in total flux.

\section{How much was needed?}

The actual chemical conditions of the origin of life are unclear and presently unknowable. However, the requirements for prebiotic reactions have been elucidated from prebiotic chemists working to promote chemical reactions of potential interest to the origin of life; these experiments may provide a basis for understanding lower limits required for chemical reactivity. For instance, the self-assembly of potential prebiotic nucleobases occurs only when the concentration of individual bases exceeds 5 millimoles per liter (mM, Cafferty et al. 2013). In this reaction, nucleobases spontaneously start to self-assemble into stacked layers, formed of planar hexads of the nucleobases. The spontaneous assembly requires a concentration of $5 \mathrm{mM}$ in order "crystallize" and form this self-assembling 


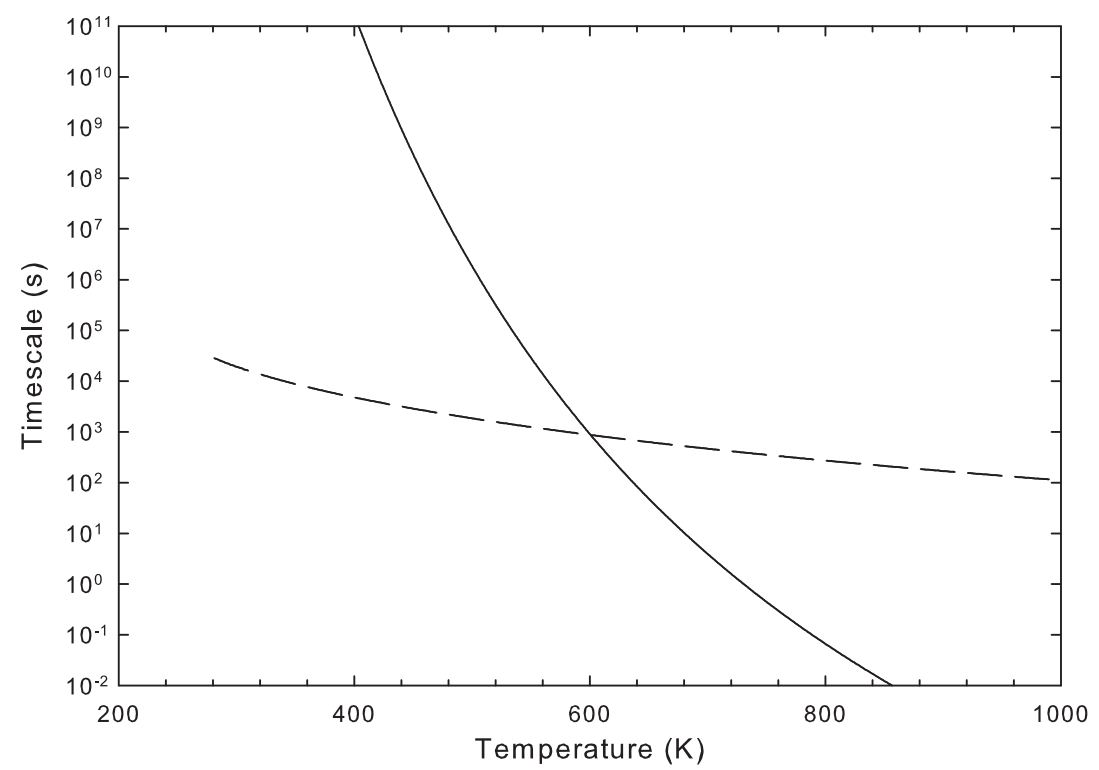

Figure 1. Timescale of energy dissipation of $60 \mathrm{MJ}$ per $\mathrm{kg}$ of a $1 \mathrm{~m}^{2}$ per $\mathrm{kg}$ object in a $220 \mathrm{~K}$ atmosphere (dashed line) compared to the timescale of degradation of 99 percent of the object's amino acids (solid line). Organics degrade rapidly above $600 \mathrm{~K}$.

structure. In reactions like these, there is an absolute minimum needed in order to promote chemical bond formation.

Other reactions, such as phosphorylation and condensation reactions, have generally required at least $10 \mathrm{mM}$ of individual reactants (Pasek and Kee 2011). In similar moleculeconstructing reactions, the construction of larger 4- and 6-carbon organic molecules from starting 2-carbon molecules provides a route to prebiotic "metabolic" organic compound construction (Butch et al. 2013). Like phosphorylation reactions, reactant concentrations were between $2 \mathrm{mM}$ to $1 \mathrm{M}$.

For most of these prebiotic reactions, concentrations of organic compounds require about $10 \mathrm{mM}$ of reagents to proceed. This value should be viewed as a target for constraining the environments where extraterrestrial prebiotic material might have accumulated sufficiently for prebiotic assembly to have proceeded, though certainly several reactions require much lower concentrations.

\section{Scenarios for accumulation}

With a global flux of about $5 \times 10^{7} \mathrm{~kg} /$ year of soluble organics during heavy bombardment periods, the total molarity of extraterrestrial organic carbon would increase by $10^{-8}$ to $10^{-9} \mathrm{mM} /$ year. Hence, extraterrestrial material is incapable of driving prebiotic chemistry in the ocean, even if organic decomposition is ignored.

The ocean is a problematic location for prebiotic chemistry due to its large size and great depth. In contrast, lakes that drain large basins are more probable environments for concentrating organic compounds. For instance, Mono Lake drains an area of about 2000 $\mathrm{km}^{2}$, and is about $3 \times 10^{12} \mathrm{~L}$ in volume. If an equivalent to Mono Lake was present on the prebiotic earth, then the concentration of organic carbon in the lake would be between 1 and $10 \mathrm{mM}$, after about $10^{6}$ years of accumulation and assuming no decomposition of the organic compounds. 
Recent work in alternative solvent chemistry has demonstrated that environments that use both water and potentially prebiotic organic solvents may overcome some of the problems of prebiotic chemistry (Gull et al. 2014, Gállego et al. 2015). These organic solvents include relatively simple molecules such as urea $\left(\mathrm{CO}\left(\mathrm{NH}_{2}\right)_{2}\right)$ and ammonium formate $\left(\mathrm{NH}_{4} \mathrm{O}_{2} \mathrm{CH}\right)$. As solutions of these materials dry, they create eutectic mixtures where reactions that normally do not happen in water (such as condensation) occur spontaneously (Gull et al. 2014). Such an environment could arise spontaneously on the earth if organic compounds accumulate in a seasonal lake during the wet season, and are solvated into the organic eutectic during the dry season. In such a scenario, concentrations of organics delivered by exogenous sources could exceed $1000 \mathrm{mM}$, perhaps sufficient to drive prebiotic chemistry.

Alternative considerations of accumulation include the potential for organics to generate new molecules upon sitting on a planet's surface. Organics within IDPs and micrometeorites pyrolyze on atmospheric entry, turning them from individual organic molecules to tar. However, recent research on the tar produced by the Miller synthesis demonstrated that amino acids are released slowly as the tar hydrolyzes (50 year timescales, see Johnson et al. 2008). By analogy, even if the amino acids decompose in IDPs as they lose energy by radiative heating, the amino acids might be recovered by the hydrolysis of the tar over geologically short timescales. This interplay between exogenous delivery and endogenous synthesis could have increased the net organic inventory of the early earth.

As a final consideration of the role of exogenous material in the origin of life, several rare materials are delivered to the earth by exogenous material. These include native iron and nickel metals, which can serve as reactive catalysts for chemical reactions such as the Fischer-Tropsch type reaction (Hill and Nuth 2003). Still another important material delivered by meteorites is the element phosphorus. Phosphorus, delivered primarily as the mineral schreibersite $(\mathrm{Fe}, \mathrm{Ni})_{3} \mathrm{P}$, is significantly more reactive in meteoritic material than in terrestrial minerals, and is capable of spontaneously phosphorylating organic molecules such as glycerol (Pasek et al. 2013), and nucleosides (Gull et al. in review). To this end, meteorites might have been quite important in providing a reactive driver for prebiotic chemistry, setting up the reactions that were critical for starting the materials of life.

\section{References}

Bottke, W. F., Vokrouhlický, D., Minton, D., Nesvorný, D., Morbidelli, A., Brasser, R., Simonson, B., \& Levison, H. F. 2012, Nature, 485, 78

Butch, C., Cope, E. D., Pollet, P., Gelbaum, L., Krishnamurthy, R., \& Liotta, C. L. 2013, J. Am. Chem. Soc., 135, 13440

Cafferty, B. J., Gállego, I., Chen, M. C., Farley, K. I., Eritja, R., \& Hud, N. V. 2013, J. Am. Chem. Soc., 135, 2447

Cleaves, H. J., Chalmers, J. H., Lazcano, A., Miller, S. L., \& Bada, J. L. 2008, Origins Life Evol. B., 38, 105

Gállego, I., Grover, M. A., \& Hud, N. V. 2015, Angew. Chem. Int. Ed., 54, 6765

Gull, M., Zhou, M., Fernández, F. M., \& Pasek, M. A. 2014, J. Molec. Evol., 78, 109

Hill, H. G. \& Nuth, J. A. 2003, Astrobiology, 3, 291

Johnson, A. P., Cleaves, H. J., Dworkin, J. P., Glavin, D. P., Lazcano, A., \& Bada, J. L. 2008, Science, 322, 404

Love, S. G. \& Brownlee, D. E. 1993, Science, 262, 550

Miller, S. L. 1953, Science, 117, 528

Pasek, M. A., Harnmeijer, J. P., Buick, R., Gull, M., \& Atlas, Z. 2013, Proc. Natl. Acad. Sci. USA, 110, 10089 
Pasek, M. A. \& Kee, T. P. 2011, in: R. Egel, D.-H. Lankenau,\& A. Y. Mulkidjanian (eds.), Origins of Life: The Primal Self-Organization (Berlin and Heidelberg: Springer), p. 57

Pasek, M. A. \& Lauretta, D. S. 2008, Origins Life Evol. B., 38, 5

Yablokov, V. A., Smel'tsova, I. L. \& Faerman, V. I. 2013, Russ. J. Gen. Chem., 83, 476

Zahnle, K. \& Grinspoon, D. 1990, Nature, 339, 463

Zhao, M. \& Bada, J. L. 1989, Nature, 339, 463 